\title{
Data mapping to augment dietary intake values from a nutritional database to a national cohort survey: protocols to improve quality of reported food intake
}

\author{
M. Crowe*1, M. O’Sullivan ${ }^{1}$, O. Cassetti ${ }^{1}$, C. McGrath ${ }^{2}$ and A. O' Sullivan ${ }^{3}$ \\ ${ }^{1}$ Dublin Dental University Hospital, Trinity College Dublin, ${ }^{2}$ Faculty of Dentistry, Hong Kong University and ${ }^{3}$ Institute \\ of Food and Health, University College Dublin
}

The Growing Up in Ireland (GUI) infant cohort was investigated to determine associations between dietary intake, dental problems and anthropometric measurements. Dietary intake is commonly measured using Food Frequency Questionnaires (FFQ). However the FFQ from the GUI survey provided a limited list of "healthy" and "unhealthy foods" and was also limited in terms of the consumption frequency recorded. This study explored the augmentation of this limited dietary intake by unidirectional mapping from the more detailed National Preschool Nutrition Survey (NPNS) which used a 4 day weighed food diary ${ }^{(1)}$. The objectives were: 1) to assess the degree to which the databases could be matched, 2) quantify the dietary food intake that was not covered by the FFQ in GUI for a similar population in terms of frequency and amount of consumption and 3) assess the lack of capture of potentially cariogenic or obesogenic foods by using a short FFQ to determine associations with health outcomes such as dental problems or anthropometric status. Data were derived from the second wave of the GUI infant cohort $(n=9,793)$ and NPNS $(n=125)$ of 3 year old children, both sampled in 2010/2011. All of the GUI FFQ categories were mapped in one direction only with food groups from NPNS. Other variables from NPNS such as BMI (Body Mass Index) classification ${ }^{(2)}$, social class and gender were also included. The population samples were assessed for difference using the two proportion $\mathrm{z}$ - test, $\mathrm{p}<0.05$.



Fig. 1. Number not covered by GUI

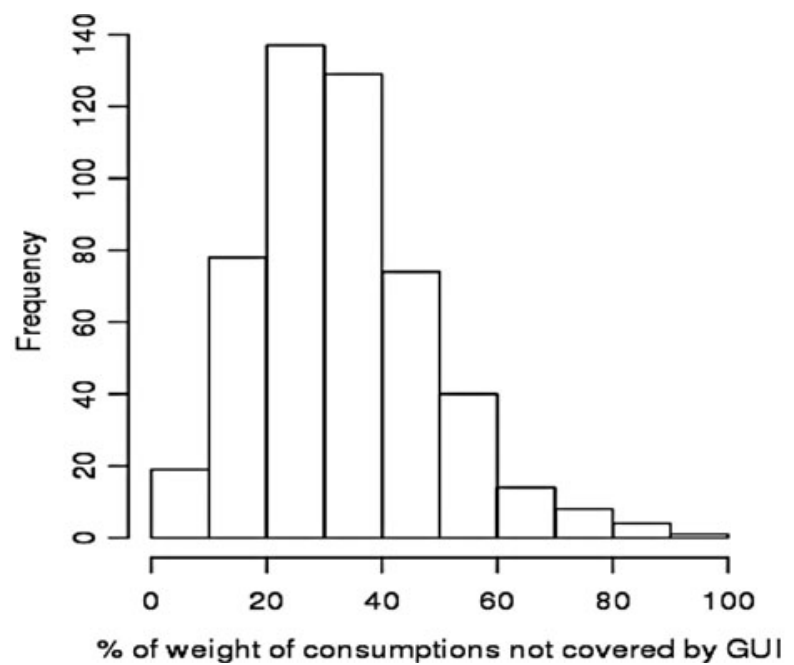

Fig. 2. Weight not covered by GUI

Both samples were nationally representative although NPNS had a higher proportion from the 'Professional' social class. The frequency of consumption of all mapped food groups varied considerably. Approximately $43 \%(\mathrm{SD}=13)$ of the food consumption occasions and approximately $33 \%(\mathrm{SD}=15)$ of the weight of food consumed in the NPNS survey were not covered by the GUI FFO categories. Potentially cariogenic foods such as fruit juices, smoothies, RTEBC, puddings and added sugar were not covered by the GUI FFQ and, on average, each of these constituted approximately $6 \%$ frequency of consumptions overall. Using a limited FFQ in large national surveys restricts the investigation of potentially associated health outcomes. This research demonstrates that if a FFQ provides insufficient dietary information for health outcome analysis, it is possible to augment dietary intake values by data-mapping. This provides more detailed information on food consumption patterns with the potential to enhance health outcome analysis. 